\title{
Changes in Alanine Aminotransferase/ Aspartate Aminotransferase Ratio Are Associated with Changes in Insulin Resistance in Japanese Community-Dwelling Middle-Aged and Elderly Women
}

\author{
Ryuichi Kawamoto ${ }^{1,2}$, Daisuke Ninomiya1,2, Yoshihisa Kasai2, Tomo Kusunoki², \\ Nobuyuki Ohtsuka², Teru Kumagi ${ }^{1}$, Masanori Abe ${ }^{1}$ \\ ${ }^{1}$ Department of Community Medicine, Ehime University Graduate School of Medicine, Ehime, Japan \\ ${ }^{2}$ Department of Internal Medicine, Seiyo Municipal Nomura Hospital, Ehime, Japan \\ Email: *rykawamo@m.ehime-u.ac.jp,98065dn@jichi.ac.jp, tbbyw2svgb@yahoo.co.jp, \\ kusunoki.tomo.mm@ehime-u.ac.jp, ohtsukan@hotmail.com, terukuma@m.ehime-u.ac.jp, \\ masaben@m.ehime-u.ac.jp
}

Received 14 May 2016; accepted 12 June 2016; published 15 June 2016

Copyright (C) 2016 by authors and Scientific Research Publishing Inc.

This work is licensed under the Creative Commons Attribution International License (CC BY). http://creativecommons.org/licenses/by/4.0/

(c) (i) Open Access

\section{Abstract}

Liver marker \{e.g., alanine aminotransferase (ALT) and aspartate aminotransferase (AST)\} levels independently predict insulin resistance. The aim of the present study is to examine how changes in liver markers are associated with changes in insulin resistance after exercise in Japanese community-dwelling adults. The participants were 76 women aged $67 \pm 6$ years from a rural village. Nordic walk (NW) exercise of 120 min per week was performed for 12 weeks. Before and at the end of the 12-week intervention, various confounding factors and insulin resistance \{e.g., Homeostatic Model Assessment of Insulin Resistance (HOMA-IR)\} were measured. The baseline, follow-up, and changes in ALT $/ A S T$ ratio $(\beta=0.390, P<0.001 ; \beta=0.393, P<0.001$; and $\beta=0.321, P=0.004$, respectively) were each significantly and independently associated with HOMA-IR. When the data were further stratified by baseline and change in ASL/AST ratio, changes in HOMA-IR decreased more significantly in participants with baseline ASL/AST ratio $\geq 0.762$ and change in ALT/AST ratio of $<0$ than those with change in ALT/AST ratio of $\geq 0$ (baseline ASL/AST ratio $<0.762, \mathrm{P}=0.002$ and ASL/AST ratio $\geq 0.762, P=0.047$ ). This study is of interest because liver transaminase markers, which are inexpensive and routinely collected in clinical settings, may provide a simple 
and accurate enhancement to models currently used to identify subjects with changes in insulin resistance. These results suggest that a higher baseline and decreased change in ALT/AST ratio may be a predictor for decreased insulin resistance after a 12-week walking exercise in community-dwelling middle-aged and elderly women.

\section{Keywords}

\section{ALT/AST Ratio, Insulin Resistance, HOMA-IR, Change, Walking Exercise, Women}

\section{Introduction}

Insulin resistance plays a central role in the pathogenesis of incident diabetes, hypertension [1], dyslipidemia [2], and cardiovascular disease (CVD) [1]-[5]. It is important for us to evaluate insulin resistance as a surrogate maker. While, accurate measurement of insulin resistance requires the use of diffuse techniques (e.g., the glucose clamp technique) that require expense and time. Thus, alternatives have been sought to simplify the measurement of insulin resistance, and the homeostatic model assessment (HOMA) [6], fasting glucose/insulin ratio (FGIR) [7] and quantitative insulin sensitivity check index (QUICKI) methods [8] have been the most frequently used techniques in clinical investigations. In particular, the HOMA approach has been widely used in clinical research to assess insulin sensitivity, and correlates reasonably with the results of clamping studies. However, the use of HOMA-IR is not readily available because insulin levels are not measured during annual health examinations and in clinical practice.

The liver is the site of glycogen synthesis, gluconeogenesis, and insulin degradation, and the liver plays a major role in the homeostasis of insulin and plasma glucose. Many studies have demonstrated that alanine aminotransferase (ALT), aspartate aminotransferase (AST) and gamma-glutamyl transferase (GGT) levels as liver markers, independently predict type 2 diabetes [9] [10], metabolic syndrome [11]-[13], and CVD [14] [15]. These markers have been shown to be associated with indirect measurements of insulin resistance including fasting insulin levels [13] [16] and HOMA-IR [9] [17]-[19]. However, there are few studies that demonstrate a relationship between changes in liver markers and insulin resistance after exercise. It is useful for us to be able to evaluate insulin resistance by measuring liver markers because they are inexpensive and routinely measured in clinical settings.

We hypothesized that changes in liver markers were associated with changes in insulin resistance after exercise. To address this hypothesis, we investigated whether changes in ALT/AST ratio and GGT were associated with changes in HOMA-IR, by a 12-week walking exercise among Japanese community-dwelling middle-aged and elderly women.

\section{Materials and Methods}

\subsection{Subjects}

The present study was designed as part of the Nomura study [20]. The study population was selected through a community-based annual check-up process from the Nomura health and welfare center in a rural town located in Ehime prefecture, Japan. Participants were enrolled in the study by public health nurses at the health and welfare center. The physical activity level of subjects, information on medical history, present conditions, and medications were obtained by interview. Candidates with CVDs or any other major illnesses that could affect the laboratory test results were excluded. All individuals aged in the range of $53-82$ years and with a clinically documented diagnosis of hypertension, dyslipidemia, type 2 diabetes, obesity, or any combination thereof were identified from the case records. The study complies with the Declaration of Helsinki, and was approved by the ethics committee of Ehime University School of Medicine (UMIN000010611) with written informed consent obtained from each subject.

\subsection{Evaluation of Risk Factors}

Information on demographic characteristics and risk factors was collected using the clinical files at baseline and 
at completion of the 12 weeks of training. Body mass index (BMI) was calculated by dividing weight (in kilograms) by the square of the height (in meters). We measured blood pressure with an appropriate-sized cuff on the right upper arm of the subjects in the sedentary position using an automatic oscillometric blood pressure recorder while they were seated after having rested for at least $5 \mathrm{~min}$. The mean of two consecutive measurements was used for analysis. Triglycerides (TG), high-density lipoprotein cholesterol (HDL-C), low-density lipoprotein cholesterol (LDL-C), ALT, AST, GGT, high sensitivity C-reactive protein (hsCRP), fasting plasma glucose (FPG), serum uric acid (SUA), and imunoreactive insulin (IRI) were measured during an overnight fast of more than 11 hours. HOMA-IR was calculated from FPG and IRI levels using the following formula; [FPG (mg/dL) $\mathrm{X}$ IRI $(\mathrm{mU} / \mathrm{mL})] / 405[6]$.

\subsection{Intervention}

Participants were required to take part in three instructor led sessions per week, each lasting 120-minute, for 12 weeks. Participants were issued poles and tutored on the correct technique for using the equipment during the first week, dedicating 120-minute sessions to the Nordic walking technique. The Nordic walking technique is a simple enhancement of normal arm swing when walking. The poles remain behind the body and pointing diagonally backward at all times. The pole length used for the Nordic walk was selected and adjusted to permit smooth arm motion, based on the INWA formula $(0.68 \times$ body height $($ in $\mathrm{cm}))$ [21], and to induce a near rightangle elbow flexion upon pole landing [22]. Assessment of post intervention dependent variables was performed within one week of the final walking session. Before and at the end of the 12-week intervention, functional tests and metabolic profiles were measured.

\subsection{Statistical Analysis}

Data are presented as the mean \pm standard deviation (SD) unless otherwise specified, and in the cases of parameters with non-normal distributions (TG, hsCRP, and HOMA-IR) the data are shown as median (interquartile range) values. In all analyses, parameters with non-normal distributions were used after log-transformation. Statistical analysis was performed using IBM SPSS Statistics Version 21 (Statistical Package for Social Science Japan, Inc., Tokyo, Japan). Twelve-week changes in various factors were calculated by subtracting the baseline values from the 12-week values. Differences among baseline and follow-up data were analyzed by paired t-test. Pearson's correlation coefficient and multiple linear regression analysis were used to estimate changes in characteristics and HOMA-IR. Furthermore, we examined the change in HOMA-IR after the 12-week Nordic walking exercise of participants stratified by baseline and change in ASL/AST ratio. A P-value $<0.05$ was considered significant.

\section{Results}

A total of 76 participants were enrolled (mean age: $67 \pm 6$ years; range, $53-81$ years) and completed the 12-week NW exercise and health examination. The prevalence of antihypertensive medication, lipid-lowering medication, and anti-diabetic medication was $34.2 \%, 21.1 \%$, and $18.4 \%$, respectively.

Baseline and follow-up characteristics of participants are shown in Table 1. Participants had a mean BMI of $24.3 \pm 3.1 \mathrm{~kg} / \mathrm{m}^{2}$ (range, $18.1-33.4 \mathrm{~kg} / \mathrm{m}^{2}$ ) and a mean waist circumference of $85.7 \pm 8.9 \mathrm{~cm}(65.5-108 \mathrm{~cm})$. Participants had several cardiovascular risk factors, and baseline BMI, waist circumference, and LDL-C were at the high end of the normal ranges. After the 12-week training exercise, BMI $(\mathrm{P}<0.001)$, waist circumference $(\mathrm{P}<$ 0.001), HDL-C $(\mathrm{P}=0.009)$, and HOMA-IR $(\mathrm{P}=0.001)$ decreased significantly. LDL-C, ALT, AST, ALT/AST ratio, GGT, TG, LDL-C, hsCRP, and SUA were unchanged.

In Table 2, we showed the relationship between baseline characteristics and HOMA-IR. In the baseline and follow-up, BMI, waist circumference, TG, HDL-C, ALT, ALT/AST ratio, GGT and serum UA correlated with HOMA-IR, but the other characteristics showed absolutely no correlation. With regard to change, change in BMI, TG, ALT, ALT/AST ratio GGT, and SUA related significantly to change in HOMA-IR.

Figure 1 shows the relationships between ALT/AST ratio and HOMA-IR. Baseline $(r=0.556, P<0.001)$, follow-up $(r=0.328, P=0.004)$, and change in ALT/AST ratio $(r=0.343, P=0.002)$ correlated significantly with each HOMA-IR. 
Table 1. Baseline and follow-up characteristics of the participants.

\begin{tabular}{|c|c|c|c|c|}
\hline Characteristics & Baseline & Follow-up & Change $(\triangle)$ & P-value* \\
\hline Body mass index $\dagger\left(\mathrm{kg} / \mathrm{m}^{2}\right)$ & $24.3 \pm 3.1$ & $23.3 \pm 2.8$ & $-0.9 \pm 0.8$ & $<0.001$ \\
\hline Waist circumference $(\mathrm{cm})$ & $85.7 \pm 8.9$ & $83.4 \pm 8.0$ & $-2.2 \pm 4.2$ & $<0.001$ \\
\hline Triglycerides (mg/dL) & $94(64-122)$ & $93(66-117)$ & $-2 \pm 40$ & 0.688 \\
\hline HDL cholesterol (mg/dL) & $69 \pm 14$ & $67 \pm 15$ & $-2 \pm 7$ & 0.009 \\
\hline LDL cholesterol (mg/dL) & $133 \pm 29$ & $130 \pm 30$ & $-3 \pm 22$ & 0.266 \\
\hline Alanine aminotransferase (IU/L) & $16(13-21)$ & $18(14-22)$ & $0 \pm 8$ & 0.608 \\
\hline Aspartate aminotransferase (IU/L) & $22(19-24)$ & $22(19-26)$ & $-1 \pm 5$ & 0.259 \\
\hline ALT/AST ratio & $0.80 \pm 0.20$ & $0.80 \pm 0.18$ & $-0.00 \pm 0.15$ & 0.965 \\
\hline Gamma-glutamyl transferase (IU/L) & $17(12-23)$ & $17(13-23)$ & $0 \pm 8$ & 0.596 \\
\hline High sensitivity CRP (mg/dL) & $0.060(0.030-0.110)$ & $0.040(0.020-0.088)$ & $0.003 \pm 0.134$ & 0.109 \\
\hline Serum uric acid (mg/dL) & $4.8 \pm 1.0$ & $4.7 \pm 1.1$ & $0.0 \pm 0.6$ & 0.519 \\
\hline HOMA-IR & $1.16(0.78-2.11)$ & $0.97(0.66-1.51)$ & $-3.8 \pm 14.4$ & 0.001 \\
\hline
\end{tabular}

Change $(\triangle)$, follow-up - baseline data. HDL, high-density lipoprotein; LDL, low density lipoprotein; ALT, Alanine aminotransferase; AST, Aspartate aminotransferase; CPR, C-reactive protein. Data presented are the mean \pm standard deviation. Data for triglycerides and high sensitivity CRP were skewed, and are presented as median (interquartile range) values, and were log-transformed for analysis. ${ }^{*} \mathrm{P}$-value from a paired t-test.

\section{Table 2. Simple relationships between participants' characteristics and HOMA-IR at each time point.}

\begin{tabular}{cccc}
\hline & & HOMA-IR & Change \\
\cline { 2 - 4 } Characteristics & Baseline & Follow-up & r (P-value) \\
\hline Body mass index & $\mathbf{r}$ (P-value) & $\mathbf{r}$ (P-value) & $0.317(0.005)$ \\
Waist circumference & $0.522(<0.001)$ & $0.450(<0.001)$ & $0.224(0.052)$ \\
Triglycerides & $0.513(<0.001)$ & $0.507(<0.001)$ & $0.323(0.004)$ \\
HDL cholesterol & $0.454(<0.001)$ & $0.453(0.004)$ & $0.102(0.383)$ \\
LDL cholesterol & $-0.371(0.001)$ & $-0.326(0.012)$ & $0.055(0.637)$ \\
Alanine aminotransferase & $0.070(0.550)$ & $0.096(0.409)$ & $0.305(0.007)$ \\
Aspartate aminotransferase & $0.437(<0.001)$ & $0.300(0.008)$ & $0.173(0.136)$ \\
ALT/AST ratio & $0.183(0.113)$ & $0.177(0.125)$ & $0.343(0.002)$ \\
Gamma-glutamyl transferase & $0.556(<0.001)$ & $0.328(0.004)$ & $0.278(0.015)$ \\
High sensitivity CRP & $0.365(0.001)$ & $0.261(0.023)$ & $0.109(0.348)$ \\
Serum uric acid & $0.059(0.614)$ & $0.161(0.164)$ & $0.238(0.038)$ \\
\hline
\end{tabular}

r, Pearson's correlation coefficient. Data for HOMA-IR, triglycerides, gamma-glutamyl transferase, and high sensitivity C-reactive protein were skewed and log-transformed for analysis.

With regard to finding independent confounding factors for each HOMA-IR, Table 3 shows the independent relationship between characteristics and HOMA-IR. Baseline BMI, TG, and ALT/AST ratio were independently associated with baseline HOMA-IR, and follow-up waist circumference, TG, ALT/AST ratio, hsCRP, and SUA were independently associated with follow-up HOMA-IR. In addition, changes in TG, ALT/AST ratio, and SUA were independently associated with changes in HOMA-IR. 

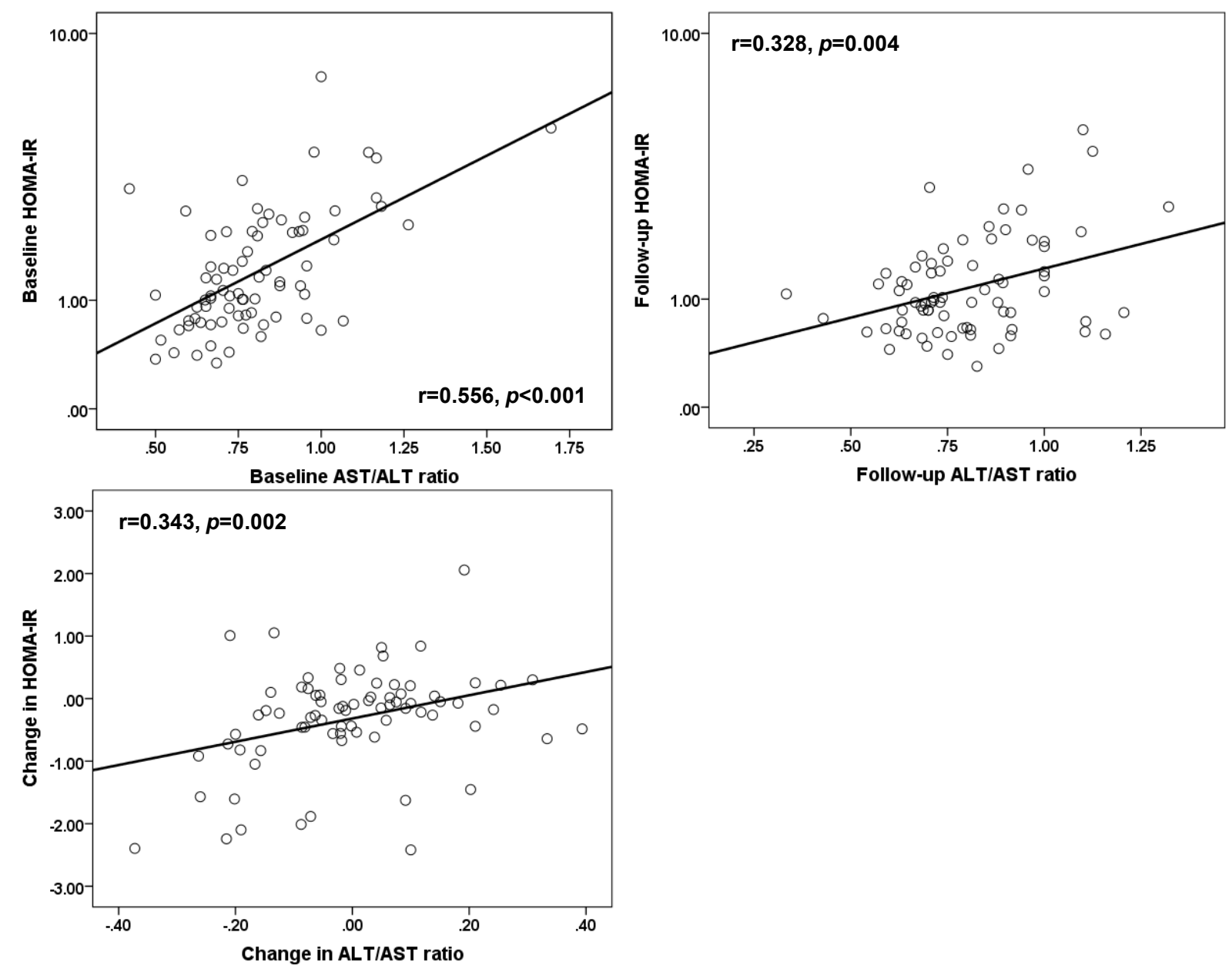

Figure 1. Relationship between ALT/AST ratio and HOMA-IR at baseline, follow-up, and the change after a 12-week Nordic walking exercise. Both baseline and follow-up HOMA-IR increased significantly with each elevated ALT/AST ratio $(\mathrm{r}=$ $0.556, \mathrm{P}<0.001 ; \mathrm{r}=0.328, \mathrm{P}=0.004$, respectively). Moreover, decreased changes in HOMA-IR correlated significantly with improved changes in ALT-AST ratio $(r=0.343, \mathrm{P}=0.002)$.

Table 3. Multivariate relationships between participants' characteristics and HOMA-IR at each time point.

\begin{tabular}{|c|c|c|c|c|}
\hline & & \multicolumn{3}{|c|}{ HOMA-IR } \\
\hline & & Baseline & Follow-up & Change \\
\hline Change in characteristics & $N=76$ & $\beta$ (P-value $)$ & $\beta$ (P-value $\left.{ }^{*}\right)$ & $\beta$ (P-value $)$ \\
\hline Body mass index & & $0.320(0.001)$ & ---- & ----- \\
\hline Waist circumference & & ----- & $0.364(<0.001)$ & ----- \\
\hline Triglycerides & & $0.228(0.015)$ & $0.351(<0.001)$ & $0.219(0.044)$ \\
\hline ALT/AST ratio & & $0.390(<0.001)$ & $0.393(<0.001)$ & $0.321(0.004)$ \\
\hline High sensitivity CRP & & ----- & $0.190(0.024)$ & ----- \\
\hline Serum uric acid & & ---- & $0.218(0.011)$ & $0.253(0.018)$ \\
\hline $\mathrm{R}^{2}$ & & $0.488(<0.001)$ & $0.556(<0.001)$ & $0.241(<0.001)$ \\
\hline
\end{tabular}

$\mathrm{r}$, Pearson's correlation coefficient. $\beta$, standardized coefficient, $\mathrm{R}^{2}$, coefficient of determination. ${ }^{*}$ Adjusted for all confounding factors in Table 2. (----), alanine aminotransferase, aspartate aminotransferase, HDL cholesterol, LDL cholesterol, and Gamma-glutamyl transferase did not remain in the final model by stepwise multiple linear regression analysis. 
When the data was further stratified by baseline and change in ASL/AST ratio, changes in HOMA-IR decreased significantly in participants with baseline ASL/AST ratio $\geq 0.762$ and change in ALT/AST ratio of $<0$ compared to those with change in ALT/AST ratio of $\geq 0$ (baseline ASL/AST ratio $<0.762, \mathrm{P}=0.002$ and baseline ASL/AST ratio $\geq 0.762, \mathrm{P}=0.047$ ) (Figure 2).

\section{Discussion}

This study demonstrated that a 12-week structured Nordic walking exercise program had a positive effect on the metabolic state of the participants. After the 12-week walking exercise, BMI, waist circumference, and HOMA-IR decreased significantly. While, ALT, AST, ALT/AST ratio, and GGT were unchanged, but the decreased change in ALT/AST ratio was significantly and independently associated with decreased change in HOMA-IR. Moreover, both higher baseline ASL/AST ratio $(\geq 0.762)$ and decreased change in ALT/AST ratio $(<0)$ were significant predictors of decreased change in HOMA-IR. We previously demonstrated that ALT/AST ratio is the best surrogate marker for insulin resistance in non-obese Japanese adults [23]. However, to our knowledge, this is the first study to have indicated these associations of changes in ALT/AST ratio with changes in HOMA-IR after a walking exercise in community-dwelling middle-aged and elderly women.

The mechanisms that lead to changes in HOMA-IR in individuals with changes in ALT/AST ratio after the 12-week walking exercise remain unclear. Nanji et al. [24] reported a significant correlation between ALT/AST ratio and the degree of fatty infiltration of the liver. It has been found that fat accumulation in the liver is characterized by insulin resistance in normal weight and moderately overweight subjects, independent of BMI and intraabdominal and overall obesity [25]. In addition, intrahepatic TG content is the best predictor of insulin action in liver, skeletal muscle, and adipose tissue, independent of BMI and percent body fat, and accounts for $34 \%, 42 \%$, and $44 \%$ of the variability in these tissues, respectively ( $\mathrm{P}<0.001$ for each model) [26]. In a crosssectional study, we have demonstrated that the optimal cut-off point in identifying insulin resistance for these markers yielded the following values: ALT/AST ratio of $\geq 0.82$ in non-obese subjects and $\geq 1.02$ in overweight subjects in Japanese community-dwelling adults [23]. Sookoian et al. have suggested that changes in transaminase levels may precede the development of a fatty liver and be caused by increased hepatic transamination of amino acids in the liver especially glutamate [13]. In this present study, we prospectively demonstrated that baseline and change in ALT/AST ratio could be markers of change in HOMA-IR after exercise training. This study is of interest because liver transaminase markers, which are inexpensive and routinely collected in clinical settings, may provide a simple and accurate enhancement to models currently used to identify subjects with changes in insulin resistance.

Some limitations of this study must be considered. First, a 12 -week intervention may potentially be too short

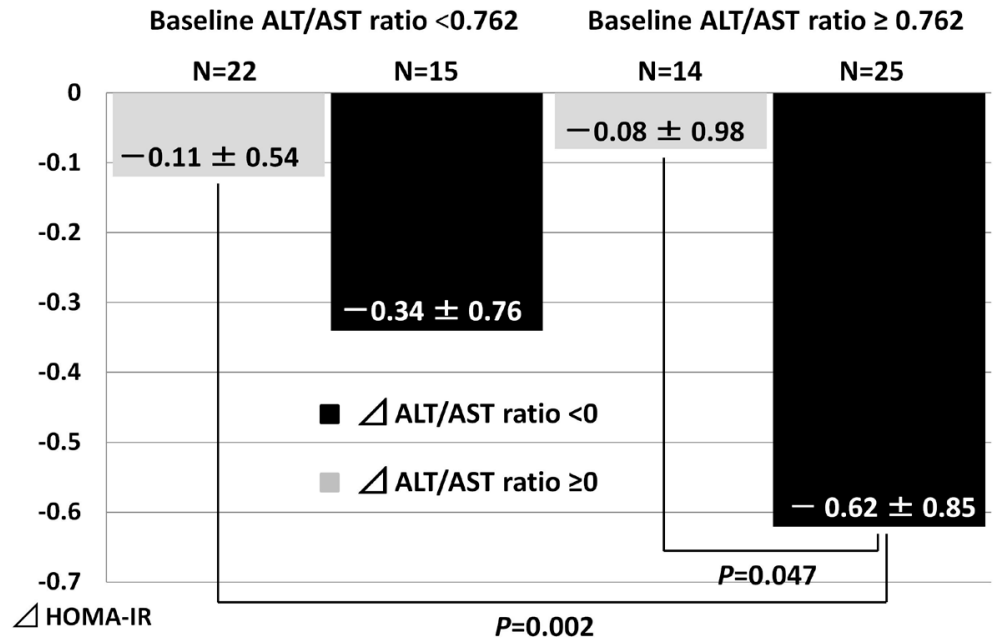

Figure 2. Change in HOMA-IR after a 12-week Nordic walking exercise of participants categorized by baseline and changes in ASL/AST ratio. Change in HOMA-IR decreased significantly in participants with baseline ASL/AST ratio $\geq 0.762$ and a change in ALT/AST ratio of $<0$ compared to those with a change in ALT/AST ratio of $\geq 0$ (baseline ASL/AST ratio $<$ $0.762, \mathrm{P}=0.002$ and baseline ASL/AST ratio $\geq 0.762, \mathrm{P}=0.047$ ). 
for measuring the relationship between AST/ALT ratio and HOMA-IR. However, changes after shorter training periods are as important as those after longer ones, because those physiological changes may attenuate after the 2nd and 3rd weeks of training. Second, we could not eliminate potential causal relationships between AST/ALT ratio and HOMA-IR because this study is not a randomized study and daily physical activity of participants, prior to entry and during the study, was not controlled or matched. Third, though we have comprehensively adjusted for confounders such as BMI, waist circumference, TG, HDL-C, LDL-C, markers of inflammation (e.g., hsCRP), and uric acid in the association of changes in AST/ALT ratio and HOMA-IR, other important measures (such as hormones and other metabolism) are absent. Fourth, we could not eliminate possible effects of underlying diseases on the results. Fifth, the number of participants may be too small to find a significant difference. Due to these weak points of this study, the demographics and referral source may limit generalizability.

\section{Conclusion}

The present study demonstrates that baseline and change in ALT/AST ratio are predictors for change in HOMA-IR after a 12-week walking exercise in community-dwelling middle-aged and elderly women. Further studies are needed to verify whether interventions (such as Nordic walking exercise) aimed at reducing ALT/AST ratio could be investigated for the prevention of CVD in a larger number of community-dwelling persons.

\section{Acknowledgements}

This work was supported in part by a grant-in-aid for Scientific Research from the Foundation for Development of Community (2015).

\section{Conflict of Interests}

The authors declare that they have no competing interests.

\section{References}

[1] Sung, K.C., Lim, S. and Rosenson, R.S. (2011) Hyperinsulinemia and Homeostasis Model Assessment of Insulin Resistance as Predictors of Hypertension: A 5-Year Follow-Up Study of Korean Sample. American Journal of Hypertension, 24, 1041-1045. http://dx.doi.org/10.1038/ajh.2011.89

[2] Jung, U.J. and Choi, M.S. (2014) Obesity and Its Metabolic Complications: The Role of Adipokines and the Relationship between Obesity, Inflammation, Insulin Resistance, Dyslipidemia and Nonalcoholic Fatty Liver Disease. International Journal of Molecular Sciences, 15, 6184-6223. http://dx.doi.org/10.3390/ijms15046184

[3] Nakamura, K., Sakurai, M., Miura, K., Morikawa, Y., Ishizaki, M., Yoshita, K., Kido, T., Naruse, Y. and Nakagawa, H. (2010) Homeostasis Model Assessment of Insulin Resistance and the Risk of Cardiovascular Events in MiddleAged Non-Diabetic Japanese Men. Diabetologia, 53, 1894-1902. http://dx.doi.org/10.1007/s00125-010-1803-Z

[4] Gotoh, S., Doi, Y., Hata, J., Ninomiya, T., Mukai, N., Fukuhara, M., Kamouchi, M., Kitazono, T. and Kiyohara, Y. (2012) Insulin Resistance and the Development of Cardiovascular Disease in a Japanese Community: The Hisayama Study. Journal of Atherosclerosis and Thrombosis, 19, 977-985. http://dx.doi.org/10.5551/jat.13698

[5] Zhang, Y., Lee, E.T., Howard, B.V., Best, L.G., Umans, J.G., Yeh, J., Wang, W., Yeh, F., Ali, T., Devereux, R.B. and de Simone, G. (2013) Insulin Resistance, Incident Cardiovascular Diseases, and Decreased Kidney Function among Nondiabetic American Indians: The Strong Heart Study. Diabetes Care, 36, 3195-3200. http://dx.doi.org/10.2337/dc12-2368

[6] Matthews, D.R., Hosker, J.P., Rudenski, A.S., Naylor, B.A., Treacher, D.F. and Turner, R.C. (1985) Homeostasis Model Assessment: Insulin Resistance and Beta-Cell Function from Fasting Plasma Glucose and Insulin Concentrations in Man. Diabetologia, 28, 412-419. http://dx.doi.org/10.1007/BF00280883

[7] Quon, M.J. (2001) Limitations of the Fasting Glucose to Insulin Ratio as an Index of Insulin Sensitivity. Journal of Clinical Endocrinology \& Metabolism, 85, 4615-4617. http://dx.doi.org/10.1210/jcem.86.10.7952

[8] Katz, A., Nambi, S.S., Mather, K., Baron, A.D., Follmann, D.A., Sullivan, G. and Quon, M.J. (2000) Quantitative Insulin Sensitivity Check Index: A Simple, Accurate Method for Assessing Insulin Sensitivity in Humans. Journal of Clinical Endocrinology \& Metabolism, 85, 2402-2410. http://dx.doi.org/10.1210/jcem.85.7.6661

[9] Gao, F., Pan, J.M., Hou, X.H., Fang, Q.C., Lu, H.J., Tang, J.L., Gu, H.L., Pan, Z.J., Yao, Y.H., Shen, W.Z. and Jia, W.P. (2012) Liver Enzyme Concentrations Are Closely Related to Prediabetes: Findings of the Shanghai Diabetes Study II (SHDS II). Biomedical and Environmental Sciences, 25, 30-37. 
[10] Ahn, H.R., Shin, M.H., Nam, H.S., Park, K.S., Lee, Y.H., Jeong, S.K., Choi, J.S. and Kweon, S.S. (2014) The Association between Liver Enzymes and Risk of Type 2 Diabetes: The Namwon Study. Diabetology \& Metabolic Syndrome, 6, 14. http://dx.doi.org/10.1186/1758-5996-6-14

[11] Steinvil, A., Shapira, I., Ben-Bassat, O.K., Cohen, M., Vered, Y., Berliner, S. and Rogowski, O. (2010) The Association of Higher Levels of Within-Normal-Limits Liver Enzymes and the Prevalence of the Metabolic Syndrome. Cardiovascular Diabetology, 9, 30. http://dx.doi.org/10.1186/1475-2840-9-30

[12] Xu, Y., Bi, Y.F., Xu, M., Huang, Y., Lu, W.Y., Gu, Y.F., Ning, G. and Li, X.Y. (2011) Cross-Sectional and Longitudinal Association of Serum Alanine Aminotransaminase and $\gamma$-Glutamyltransferase with Metabolic Syndrome in Middle-Aged and Elderly Chinese People. Journal of Diabetes, 3, 38-47. http://dx.doi.org/10.1111/j.1753-0407.2010.00111.x

[13] Sookoian, S. and Pirola, C.J. (2012) Alanine and Aspartate Aminotransferase and Glutamine-Cycling Pathway: Their Roles in Pathogenesis of Metabolic Syndrome. World Journal of Gastroenterology, 18, 3775-3781. http://dx.doi.org/10.3748/wjg.v18.i29.3775

[14] Fraser, A., Harris, R., Sattar, N., Ebrahim, S., Davey Smith, G. and Lawlor, D.A. (2007) Gamma-Glutamyltransferase Is Associated with Incident Vascular Events Independently of Alcohol Intake: Analysis of the British Women's Heart and Health Study and Meta-Analysis. Arteriosclerosis, Thrombosis, and Vascular Biology, 27, 2729-2735. http://dx.doi.org/10.1161/ATVBAHA.107.152298

[15] Lee, D.H., Buijsse, B., Steffen, L., Holtzman, J., Luepker, R. and Jacobs Jr., D.R. (2009) Association between Serum Gamma-Glutamyltransferase and Cardiovascular Mortality Varies by Age: The Minnesota Heart SURVEY. European Journal of Preventive Cardiology, 16, 16-20. http://dx.doi.org/10.1097/HJR.0b013e32830aba5c

[16] Saleh, J., Farhan, H., Al-Saqri, I., Al-Riyami, B. and Cianflone, K. (2013) Plasma Gamma-Glutamyltransferase Is Strongly Determined by Acylation Stimulating Protein Levels Independent of Insulin Resistance in Patients with Acute Coronary Syndrome. Disease Markers, 35, 155-161.

[17] Vozarova, B., Stefan, N., Lindsay, R.S., Saremi, A., Pratley, R.E., Bogardus, C. and Tataranni, P.A. (2002) High Alanine Aminotransferase Is Associated with Decreased Hepatic Insulin Sensitivity and Predicts the Development of Type 2 Diabetes. Diabetes, 51, 1889-1895. http://dx.doi.org/10.2337/diabetes.51.6.1889

[18] Ortega, E., Koska, J., Salbe, A.D., Tataranni, P.A. and Bunt, J.C. (2006) Serum Gamma-Glutamyl Transpeptidase Is a Determinant of Insulin Resistance Independently of Adiposity in Pima Indian Children. The Journal of Clinical Endocrinology \& Metabolism, 91, 1419-1422. http://dx.doi.org/10.1210/jc.2005-1783

[19] Kawamoto, R., Tabara, Y., Kohara, K., Miki, T., Kusunoki, T., Takayama, S., Abe, M., Katoh, T. and Ohtsuka, N. (2010) High-Sensitivity C-Reactive Protein and Gamma-Glutamyl Transferase Levels Are Synergistically Associated with Metabolic Syndrome in Community-Dwelling Persons. Cardiovascular Diabetology, 9, 87. http://dx.doi.org/10.1186/1475-2840-9-87

[20] Kawamoto, R., Kohara, K., Katoh, T., Kusunoki, T., Ohtsuka, N., Abe, M., Kumagi, T. and Miki, T. (2015) Changes in Oxidized Low-Density Lipoprotein Cholesterol Are Associated with Changes in Handgrip Strength in Japanese Community-Dwelling Persons. Endocrine, 48, 871-877. http://dx.doi.org/10.1007/s12020-014-0360-5

[21] International Nordic Walking Federation (INWA) (2011) INWA Nordic Walking Portal. http://www.inwa-nordicwalking.com/webcite

[22] Schwameder, H., Roithner, R., Muller, E. and Niessen, W. (1999) Knee Joint Forces during Downhill Walking with Hiking Poles. Journal of Sports Sciences, 17, 969-978. http://dx.doi.org/10.1080/026404199365362

[23] Kawamoto, R., Kohara, K., Kusunoki, T., Tabara, Y., Abe, M. and Miki, T. (2012) Alanine Aminotransferase/Aspartate Aminotransferase Ratio Is the Best Surrogate Marker for Insulin Resistance in Non-Obese Japanese Adults. Cardiovascular Diabetology, 11, 117. http://dx.doi.org/10.1186/1475-2840-11-117

[24] Nanji, A.A., French, S.W. and Freeman, J.B. (1986) Serum Alanine Aminotransferase to Aspartate Aminotransferase Ratio and Degree of Fatty Liver in Morbidly Obese Patients. Enzyme, 36, 266-269.

[25] Seppälä-Lindroos, A., Vehkavaara, S., Häkkinen, A.M., Goto, T., Westerbacka, J., Sovijärvi, A., Halavaara, J. and Yki-Järvinen, H. (2002) Fat Accumulation in the Liver Is Associated with Defects in Insulin Suppression of Glucose Production and Serum Free Fatty Acids Independent of Obesity in Normal Men. The Journal of Clinical Endocrinology \& Metabolism, 87, 3023-3328. http://dx.doi.org/10.1210/jcem.87.7.8638

[26] Korenblat, K.M., Fabbrini, E., Mohammed, B.S. and Klein, S. (2008) Liver, Muscle, and Adipose Tissue Insulin Action Is Directly Related to Intrahepatic Triglyceride Content in Obese Subjects. Gastroenterology, 134, 1369-1375. http://dx.doi.org/10.1053/j.gastro.2008.01.075 